\title{
Spatio-temporal delays in a nutrient-plankton model on a finite domain: linear stability and bifurcations
}

\author{
Stephen A. Gourley ${ }^{a, *}$, Shigui Ruan ${ }^{\text {b,1 }}$ \\ ${ }^{a}$ Department of Mathematics and Statistics, University of Surrey, Guildford, Surrey GU2 7XH, UK \\ ${ }^{\mathrm{b}}$ Department of Mathematics and Statistics, Dalhousie University, Halifax, \\ Nova Scotia, Canada B3H $3 J 5$
}

\begin{abstract}
The issue of how to incorporate time-delays into a mathematical model in which individuals are moving around requires careful consideration. Any time-delay term must also involve a weighted spatial averaging to account for movement of individuals during the time-delay period. Most of the current literature on this subject is on reaction-diffusion equations and concentrates on the simplest case when the spatial domain is infinite. In this paper we consider what changes arise when the domain is finite. Spatial averaging kernels are computed explicitly for the case of a finite, one-dimensional domain. To illustrate the ideas we concentrate on a diffusive nutrient-plankton model. The model is analysed in terms of the local stability of the steady states and bifurcations. The results of some numerical simulations are also presented.

(C) 2002 Elsevier Inc. All rights reserved.
\end{abstract}

Keywords: Non-local delay; Reaction-diffusion equations; Stability; Bifurcation; Spatio-temporal pattern

\footnotetext{
${ }^{*}$ Corresponding author.

E-mail addresses: s.gourley@surrey.ac.uk (S.A. Gourley), ruan@mathstat.dal.ca (S. Ruan).

${ }^{1}$ Research supported by the Natural Science and Engineering Research Council (NSERC) of Canada.
}

0096-3003/\$ - see front matter (C) 2002 Elsevier Inc. All rights reserved.

doi:10.1016/S0096-3003(02)00494-0 


\section{Introduction}

In recent years there has been a great deal of interest in ordinary differential equations and reaction-diffusion equations modelling the evolution of a plankton population feeding on nutrient that is supplied at a constant rate and which is partially recycled through bacterial decomposition of the dead plankton. One of the earliest mathematical models was due to Nisbet and Gurney [1] who incorporated the nutrient recycling into their model as an instantaneous term. However, in nature nutrient recycling takes time. It happens more quickly at high temperatures than at low temperatures [2] but always takes a certain amount of time, and therefore in the model equations there needs to be a time-delay to account for this. In 1990, Beretta et al. [3] proposed a chemostat-type model in which nutrient recycling was modelled by using a distributed delay term. Bischi [4] analysed the same model from the point of view of the effect of the delay on resilience.

The time-delay due to nutrient recycling is not the only delay effect present. Experiments have shown that, in addition to the delays involved in the decomposition of dead organisms, there is also a delay in the growth response of the species to nutrient uptake [5]. To account for this, Ruan [6] modified the model of Beretta et al. [3] by incorporating a discrete delay in the species' growth response, retaining also the distributed delay term used to model the nutrient recycling. Increasing the discrete delay caused the model to undergo a Hopf bifurcation to a limit cycle solution. Beretta and Takeuchi $[7,8]$ modelled both of the two delay effects using distributed delay terms and studied the global asymptotic stability of the positive equilibrium by using Lyapunov functionals. See also the works of He et al. $[9,10]$ where other related results are proved.

All of the above models are purely time dependent ODEs with delays; that is to say, spatial effects are ignored. However, in the lakes and oceans it is clear that spatial effects will be very important. Plankton can move around subject to many factors including diffusion and currents. The simplest way to incorporate diffusion of the plankton is to use reaction-diffusion equations $[11,12]$. Reaction-diffusion equations incorporating time-delays are more difficult to study although considerable progress has been made in recent years. Ruan [13] proposed and studied the following reaction-diffusion system to account for spatial dispersal of both the nutrient $N$ and the plankton $P$.

$$
\begin{aligned}
& \frac{\partial N}{\partial t}=d_{1} \nabla^{2} N+D\left(N^{0}-N\right)-a U(N) P+\gamma_{1} \int_{-\infty}^{t} f_{1}(t-s) P(x, s) \mathrm{d} s, \\
& \frac{\partial P}{\partial t}=d_{2} \nabla^{2} P+P\left[-(\gamma+D)+a_{1} \int_{-\infty}^{t} f_{2}(t-s) U(N(x, s)) \mathrm{d} s\right] .
\end{aligned}
$$

This system generalises the models described thus far by the addition of diffusion. Also, both of the two delay effects are modelled by using distributed delays. Ruan obtained results on the linear stability of the positive equilibrium 
together with theorems on the possible bifurcations from this equilibrium, which include periodic travelling waves (wave trains).

It has become recognised in recent years that, while systems such as the above may yield a great deal of insight into the role of time-delay in a diffusive model, there is a certain problem associated with simply adding diffusion terms to a time-delay ODE, or simply inserting a time-delay into a reaction-diffusion system. The problem is that, at previous times, individuals have not necessarily been at the same point in space. They could, in fact, have been anywhere in the spatial domain, since a further well-known fact about simple Fickian diffusion leading to a Laplacian term, is that it implies that the underlying propagation speed is infinite. This problem has been addressed in several papers in recent years; see Britton [14], Gourley and Britton [15], and Thieme and Zhao [16] for descriptions of how the delays may be correctly incorporated into the reactiondiffusion setting. Other papers which discuss this modelling issue, in the context of patch models, are those of Madras et al. [17] and So et al. [18].

In the reaction-diffusion setting, the research described in the above mentioned papers has shown that, on an infinite spatial domain, one can address the complication of the individuals moving around during the time-delay period by including in any delay term an integration in space or, to be more precise, a weighted spatial averaging to account for the drift of individuals to their present position from all their possible positions at previous times. The weighted spatial averaging is derived using probabilistic arguments and, on an infinite domain, one concludes that the averaging is weighted according to the fundamental solution of the heat equation, with the diffusivity chosen to match that of the species under consideration. Boushaba and Ruan [19] have demonstrated that on a large finite domain, the infinite domain approach to modelling the time-delay term also appears to work satisfactorily in an approximate sense.

But on a finite domain there is a further complication in that the individuals, as well as having been moving around during the time-delay period, may also have been interacting with the domain's boundaries. Indeed, such interactions are only to be expected especially if the domain is a small one and clearly the infinite domain formulation of the spatial averaging will break down, since it cannot account for such interactions. Our aim in this paper is to correctly derive the spatial averaging kernels for a finite domain situation (we concentrate on a one-dimensional domain for simplicity). As we shall see, the kernels (the functions $G_{1}$ and $G_{2}$ in the system (1.2) below) are again chosen to be appropriately normalised solutions of the heat equation, but are now subject to whatever boundary conditions are being applied to the problem itself (which in this paper will be of the homogeneous Neumann kind). In the one-dimensional domain we use here, the averaging kernels can still be computed explicitly. In their model, Boushaba and Ruan [19] retained the formulation of the delay term that is appropriate for the infinite domain case, and they supplemented 
their model with homogeneous Neumann boundary conditions. They had to assume their domain was a large one. Our approach works for a finite domain of any size because we derive an averaging kernel that allows for interactions with the domain's boundaries. Thus in our approach the boundary conditions are not merely conditions supplemented to the governing PDEs but also play a role in the derivation of the time-delay terms themselves.

Thus, the main aim of the present paper is to improve the models referred to thus far to include spatio-temporal delays which correctly account for the interaction of delay with diffusion in a finite domain. We shall study the system

$$
\begin{aligned}
\frac{\partial N}{\partial t}= & d_{1} \frac{\partial^{2} N}{\partial x^{2}}+D\left(N^{0}-N\right)-a U(N) P \\
& +\gamma_{1} \int_{-\infty}^{t} \int_{0}^{\pi} G_{1}(x, y, t-s) f_{1}(t-s) P(y, s) \mathrm{d} y \mathrm{~d} s, \\
\frac{\partial P}{\partial t}= & d_{2} \frac{\partial^{2} P}{\partial x^{2}}+P[-(\gamma+D) \\
& \left.+a_{1} \int_{-\infty}^{t} \int_{0}^{\pi} G_{2}(x, y, t-s) f_{2}(t-s) U(N(y, s)) \mathrm{d} y \mathrm{~d} s\right]
\end{aligned}
$$

for $x \in[0, \pi]$. All the ideas carry over to $n$-dimensions; our choice of the onedimensional domain $[0, \pi]$ is purely for notational simplicity. There is no loss of generality in having the domain being of length $\pi$ since the diffusivities $d_{1}$ and $d_{2}$ are both kept general. The equations are to be solved for $t>0$ and require initial data to be prescribed for all $t \leqslant 0$ and all $x \in[0, \pi]$. For boundary conditions, we shall consider the biologically realistic homogeneous Neumann problem, so the boundary conditions are

$$
\frac{\partial P}{\partial x}=\frac{\partial N}{\partial x}=0 \quad \text { at } x=0, \pi .
$$

We shall now explain why the time-delay terms in (1.2) assume the particular form we have taken. We discuss only the term in the first equation:

$$
\int_{-\infty}^{t} \int_{0}^{\pi} G_{1}(x, y, t-s) f_{1}(t-s) P(y, s) \mathrm{d} y \mathrm{~d} s .
$$

The distributed time-delay, the integral over $s$ from $-\infty$ to $t$, weighted by the function $f_{1}$, follows a long tradition of using distributed delays to model timelags and in this respect our formulation is consistent with the distributed timedelays found in the earlier papers described in our introductory paragraphs. The function $f_{1}(t)$ is called the delay kernel. It will always be assumed to satisfy $f_{1}(t) \geqslant 0$ for all $t \geqslant 0$ together with a normalisation condition also satisfied by $f_{2}(t)$, i.e., we assume that

$$
\int_{0}^{\infty} f_{i}(t) \mathrm{d} t=1, \quad i=1,2,
$$


which ensures that the spatially uniform steady states are unaffected by the delay. The kernel $f_{1}(t)$ is, by definition, the weight given to the population $t$ time units ago. The difference between the distributed delay terms in (1.1), and the formulation (1.3), is that the latter also involves an averaging in space, weighted by the kernel $G(x, y, t)$. This is to account for the modelling difficulties inherent in trying to incorporate time-delays into a diffusive model. At a typical time $s<t$ the density $P$ is first multiplied by the weight given to densities at this time, which is $f_{1}(t-s)$ by the way $f_{1}$ is defined. Now, the individuals which are at point $x$ at time $t$ could have been anywhere at the earlier time $s$. The idea is to calculate the probability of an individual having been at point $y$ at the earlier time $s$, given that it is at point $x$ at the current time $t$. We then sum over all possible previous positions $y$, and this explains the integral over $y$. The kernel $G_{1}$ is therefore essentially a probability density function. The detailed probabilistic arguments are explained in [14] for the case of an infinite one-dimensional domain, and in that scenario the kernel $G_{1}$ turns out to be the fundamental solution of the heat equation. In our situation the domain is finite with homogeneous Neumann boundary conditions, and the difference here is that $G_{1}$ must be chosen, in our case, to satisfy

$$
\frac{\partial G_{1}}{\partial t}=d_{2} \frac{\partial^{2} G_{1}}{\partial y^{2}}
$$

subject to

$$
\frac{\partial G_{1}}{\partial y}=0 \quad \text { at } y=0, \pi, \quad \text { and } \quad G_{1}(x, y, 0)=\delta(x-y) .
$$

The reason why the diffusivity in (1.5) has been taken as $d_{2}$ is that this is the diffusivity of the plankton $P$ which is the quantity in the delay term we are discussing. Solving the above problem, we find that

$$
G_{1}(x, y, t)=\frac{1}{\pi}+\frac{2}{\pi} \sum_{n=1}^{\infty} \mathrm{e}^{-d_{2} n^{2} t} \cos n x \cos n y .
$$

In a similar way we shall find that the spatial averaging kernel $G_{2}$ in the delay term of the second equation in (1.2) is

$$
G_{2}(x, y, t)=\frac{1}{\pi}+\frac{2}{\pi} \sum_{n=1}^{\infty} \mathrm{e}^{-d_{1} n^{2} t} \cos n x \cos n y
$$

which involves $d_{1}$, the diffusivity of the nutrient $N$.

If in (1.6) and (1.7) the $\cos n x \cos n y$ terms are replaced by

$$
\frac{1}{2}(\cos n(x+y)+\cos n(x-y))
$$


then one easily sees that each individual term in the summation in (1.6) or (1.7) is accounting for an interaction with one or more of the boundaries $x=0$ and $\pi$ of our domain $[0, \pi]$.

Throughout the whole of this paper, $G_{1}(x, y, t)$ and $G_{2}(x, y, t)$ always assume the expressions given by (1.6) and (1.7). However, unless we state otherwise, the delay kernels $f_{1}$ and $f_{2}$ are just arbitrary non-negative functions satisfying the normalisation condition (1.4). We shall aim to obtain results which hold for relatively arbitrary kernels $f_{1}$ and $f_{2}$ satisfying these conditions only, but in the literature certain particular cases are frequently considered because they facilitate analytic study and/or numerical simulation. Dropping subscripts for the moment, these cases are

$$
f(t)=\delta(t-\tau), \quad f(t)=\frac{1}{\tau} \mathrm{e}^{-t / \tau} \quad \text { and } \quad f(t)=\frac{t}{\tau^{2}} \mathrm{e}^{-t / \tau} .
$$

The first of these kernels gives rise to a model having a discrete time-delay. For example, if $f_{1}$ assumes this expression then the delay term (1.3) becomes

$$
\int_{0}^{\pi}\left\{\frac{1}{\pi}+\frac{2}{\pi} \sum_{n=1}^{\infty} \mathrm{e}^{-d_{2} n^{2} \tau} \cos n x \cos n y\right\} P(y, t-\tau) \mathrm{d} y
$$

and the idea of spatial averaging arising because of the delay becomes clearer. If $\tau$ is set to zero then the curly bracketed part becomes $\delta(x-y)$ and the whole expression simply reduces to $P(x, t)$. If $\tau$ is very small then the curly bracketed part is close to $\delta(x-y)$, reflecting the idea that most of the individuals that are at $x$ at time $t$ will not have been far away at the recent past time $t-\tau$. On the other hand if $\tau$ is very large the curly bracketed part approximates to $1 / \pi$, reflecting the idea that these individuals will have been uniformly distributed throughout the domain a long time ago.

The other two kernels in (1.8) are called weak and strong generic delay kernels. The "weak" case $f(t)=(1 / \tau) \mathrm{e}^{-t / \tau}$ reflects the idea that the importance of the past decreases exponentially the further one looks into the past. The "strong" case $f(t)=\left(t / \tau^{2}\right) \mathrm{e}^{-t / \tau}$ can be regarded as a smoothed out version of the discrete delay case $f(t)=\delta(t-\tau)$. This strong kernel has a maximum at $t=\tau$ and this means that the population density $\tau$ time units ago is more important than any other (the delta function simply singles out the density at this particular time only).

Following Macdonald [20] we introduce the average time-lags, or mean delays, which are defined as

$$
T_{f_{1}}=\int_{0}^{\infty} t f_{1}(t) \mathrm{d} t \text { and } T_{f_{2}}=\int_{0}^{\infty} t f_{2}(t) \mathrm{d} t .
$$

For the three kernels (1.8) the mean delays are $\tau, \tau$ and $2 \tau$ respectively. 
We now discuss the various parameters in (1.2). They have the following interpretations:

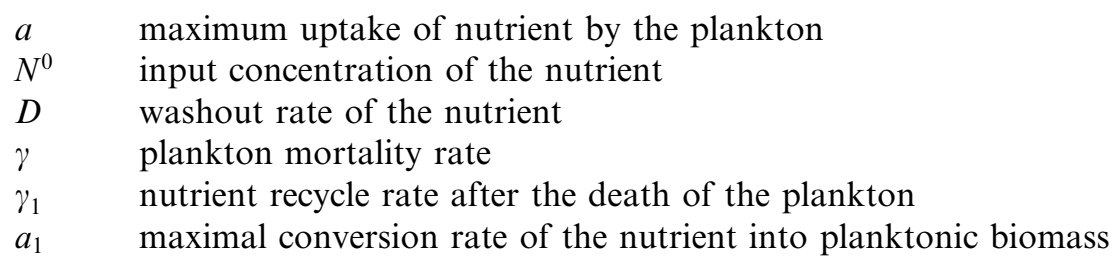

For biological realism we need to assume that

$$
\gamma_{1} \leqslant \gamma \text { and } a_{1} \leqslant a \text {. }
$$

The function $U(N)$ describes the nutrient uptake rate of plankton. It will be assumed to be a differentiable function satisfying

$$
U(0)=0, \quad \frac{\mathrm{d} U}{\mathrm{~d} N}>0 \quad \text { and } \quad \lim _{N \rightarrow \infty} U(N)=1 .
$$

We shall allow $U$ to be any function satisfying (1.12) except where otherwise stated, but a biologically reasonable choice would be the Michaelis-Menten function

$$
U(N)=\frac{N}{k+N} .
$$

In the next section we shall show how the system (1.2) may be linearised about its spatially uniform steady states and we shall obtain various results on the linear stability of the equilibria of the system. Then we shall examine under what circumstances the interior equilibrium may be driven unstable. Finally we present the results of some numerical simulations.

\section{Stability of the uniform equilibria}

First note that the spatial averaging kernels $G_{1}$ and $G_{2}$ both satisfy

$$
\int_{0}^{\pi} G_{i}(x, y, t) \mathrm{d} y=1, \quad i=1,2 .
$$

This fact, together with the fact that the delay kernels $f_{i}$ satisfy $\int_{0}^{\infty} f_{i}(t) \mathrm{d} t=1$, implies that the non-local delay terms have no effect on the spatially uniform steady state solutions. It follows that these solutions are given by $\left(N^{*}, P^{*}\right)=$ $\left(N^{0}, 0\right)$ and

$$
\left(N^{*}, P^{*}\right)=\left(U^{-1}\left(\frac{\gamma+D}{a_{1}}\right), \frac{D\left(N^{0}-N^{*}\right)}{a U\left(N^{*}\right)-\gamma_{1}}\right) .
$$

The latter is biologically relevant only if

$$
\gamma+D<a_{1} \text { and } N^{*}<N^{0} .
$$


If $\gamma+D \geqslant a_{1}$ then $\left(N^{0}, 0\right)$ is the only biologically relevant equilibrium, and it can be shown that under these circumstances $\left(N^{0}, 0\right)$ is linearly stable independently of the delays. The analysis is similar to, but considerably simpler than, the analysis for the equilibrium (2.1) because the dispersion relation arising from the linearisation about $\left(N^{0}, 0\right)$ does not involve the kernels $f_{i}$. In the remainder of this section we shall concentrate on the interior equilibrium (2.1) and we shall therefore assume henceforth that the parameters of the model satisfy (2.2), in addition to (1.11). Note that these conditions assure us that the denominator in the expression for $P^{*}$ is positive.

To investigate the linearised stability of $(2.1)$, we set $N=N^{*}+u, P=P^{*}+v$, substitute into (1.2) and retain only linear terms in $u$ and $v$, giving

$$
\begin{aligned}
\frac{\partial u}{\partial t}= & d_{1} \frac{\partial^{2} u}{\partial x^{2}}-\left(D+a P^{*} U^{\prime}\left(N^{*}\right)\right) u-a U\left(N^{*}\right) v \\
& +\gamma_{1} \int_{-\infty}^{t} \int_{0}^{\pi} G_{1}(x, y, t-s) f_{1}(t-s) v(y, s) \mathrm{d} y \mathrm{~d} s, \\
\frac{\partial v}{\partial t}= & d_{2} \frac{\partial^{2} v}{\partial x^{2}} \\
& +a_{1} P^{*} U^{\prime}\left(N^{*}\right) \int_{-\infty}^{t} \int_{0}^{\pi} G_{2}(x, y, t-s) f_{2}(t-s) u(y, s) \mathrm{d} y \mathrm{~d} s .
\end{aligned}
$$

Since the boundary conditions are homogeneous Neumann on the domain $[0, \pi]$ the appropriate trial solution is

$$
(u, v)=\left(c_{1}, c_{2}\right) \mathrm{e}^{\sigma t} \cos m x, \quad m=0,1,2, \ldots
$$

Note the effect that the non-local terms have upon such a trial solution. Dropping subscripts for the moment, we find that

$$
\int_{-\infty}^{t} \int_{0}^{\pi} G(x, y, t-s) f(t-s) \mathrm{e}^{\sigma s} \cos m y \mathrm{~d} y \mathrm{~d} s
$$

is equal to

$$
\int_{-\infty}^{t} \mathrm{e}^{\sigma s} f(t-s) \int_{0}^{\pi} \cos m y\left\{\frac{1}{\pi}+\frac{2}{\pi} \sum_{n=1}^{\infty} \mathrm{e}^{-d n^{2}(t-s)} \cos n x \cos n y\right\} \mathrm{d} y \mathrm{~d} s
$$

which, after some algebra, gives

$$
\bar{f}\left(\sigma+d m^{2}\right) \mathrm{e}^{\sigma t} \cos m x
$$

for any $m=0,1,2, \ldots$, using the orthogonality of $\{\cos m x\}_{m=0}^{\infty}$ over $[0, \pi]$. Here, $\bar{f}$ denotes the Laplace transform of $f$. Substitution of the trial solution (2.4) we find that, in order to have non-trivial solutions, $\sigma$ must satisfy $\Phi(\sigma ; m)=0$ where 


$$
\begin{aligned}
\Phi(\sigma ; m):= & {\left[\sigma+d_{1} m^{2}+D+a P^{*} U^{\prime}\left(N^{*}\right)\right]\left(\sigma+d_{2} m^{2}\right) } \\
& +a_{1} P^{*} U^{\prime}\left(N^{*}\right) \bar{f}_{2}\left(\sigma+d_{1} m^{2}\right)\left[a U\left(N^{*}\right)-\gamma_{1} \bar{f}_{1}\left(\sigma+d_{2} m^{2}\right)\right] .
\end{aligned}
$$

The equation $\Phi(\sigma ; m)=0$ is called the dispersion relation or eigenvalue equation, and the function $\Phi(\sigma ; m)$ will be referred to as the eigenvalue function. The steady state (2.1) will be linearly stable if all the roots $\sigma$ of $\Phi(\sigma ; m)=0$ are strictly in the left half of the complex plane for every integer $m=0,1,2, \ldots$.

We shall analyse the eigenvalue equation mainly by using the principle of the argument. It is, in general, a transcendental equation since $\sigma$ occurs inside the arguments of the Laplace transforms of the delay kernels. It is easily seen that the Laplace transforms $\bar{f}_{1}(\sigma)$ and $\bar{f}_{2}(\sigma)$ both converge for $\operatorname{Re} \sigma \geqslant 0$. Since Laplace transforms are analytic in their half plane of convergence, this means that the function $\Phi(\sigma ; m)$ is analytic for $\operatorname{Re} \sigma \geqslant 0$. As a consequence, a well known result in complex variable theory states that for any given $m=0,1,2, \ldots$ the number of roots of $\Phi(\sigma ; m)=0$ in the right half of the complex plane is given by the formula

$$
\lim _{R \rightarrow \infty} \frac{1}{2 \pi i} \int_{\gamma(R)} \frac{\Phi^{\prime}(\sigma ; m)}{\Phi(\sigma ; m)} \mathrm{d} \sigma
$$

where $\gamma(R)$ is the closed semicircular contour of radius $R$ that is centered at the origin and contained in $\operatorname{Re} \sigma \geqslant 0$. It can be shown, using methods similar to those described in Gourley and Bartuccelli [21], that the above integral equals

$$
1-\frac{1}{\pi} \lim _{R \rightarrow \infty} \arg \Phi(\mathrm{i} R ; m)
$$

so this is the formula we shall study. For linear stability of the uniform state (2.1) the above formula would have to yield a value 0 for each wave number $m=0,1,2, \ldots$

The use of formula (2.6) involves calculating the total change in the argument of the complex number $\Phi(\mathrm{i} R ; m)$ as its parameter $R$ goes from 0 to infinity. To compute this we need to study the graph of $\operatorname{Im} \Phi(\mathrm{i} R ; m)$ against $\operatorname{Re} \Phi(\mathrm{i} R ; m)$ for $R \in[0, \infty)$. The precise graph depends, of course, on the delay kernels $f_{1}$ and $f_{2}$ which are unspecified. However, regardless of what these kernels may be, we can show that as far as formula (2.6) is concerned the precise possibilities are all easily enumerated, and all but one of them gives us instability of the steady state (2.1). To see this, first note that we can write

$$
\bar{f}_{1}\left(\mathrm{i} R+d_{2} m^{2}\right)=\int_{0}^{\infty} f_{1}(t) \mathrm{e}^{-\left(\mathrm{i} R+d_{2} m^{2}\right) t} \mathrm{~d} t=C_{1}(R ; m)-\mathrm{i} S_{1}(R ; m)
$$

and, similarly, $\bar{f}_{2}\left(\mathrm{i} R+d_{1} m^{2}\right)=C_{2}(R ; m)-\mathrm{i} S_{2}(R ; m)$ where 


$$
\begin{aligned}
& C_{1}(R ; m)=\int_{0}^{\infty} f_{1}(t) \mathrm{e}^{-d_{2} m^{2} t} \cos R t \mathrm{~d} t \\
& S_{1}(R ; m)=\int_{0}^{\infty} f_{1}(t) \mathrm{e}^{-d_{2} m^{2} t} \sin R t \mathrm{~d} t \\
& C_{2}(R ; m)=\int_{0}^{\infty} f_{2}(t) \mathrm{e}^{-d_{1} m^{2} t} \cos R t \mathrm{~d} t \\
& S_{2}(R ; m)=\int_{0}^{\infty} f_{2}(t) \mathrm{e}^{-d_{1} m^{2} t} \sin R t \mathrm{~d} t
\end{aligned}
$$

It is obvious that

$$
\left|C_{j}(R ; m)\right| \leqslant 1 \quad \text { and } \quad\left|S_{j}(R ; m)\right| \leqslant 1
$$

for $j=1,2$ and each integer $m=0,1,2, \ldots$ Also in the proofs of certain of our theorems we shall need the following estimate, which uses the inequality $|\sin x| \leqslant x$ for $x \geqslant 0$ :

$$
\begin{aligned}
\left|S_{1}(R ; m)\right| & \leqslant \int_{0}^{\infty} f_{1}(t) \mathrm{e}^{-d_{2} m^{2} t}|\sin R t| \mathrm{d} t \leqslant \int_{0}^{\infty} f_{1}(t) \mathrm{e}^{-d_{2} m^{2} t} R t \mathrm{~d} t \\
& \leqslant R \int_{0}^{\infty} t f_{1}(t) \mathrm{d} t=R T_{f_{1}} .
\end{aligned}
$$

Similarly, we have $\left|S_{2}(R ; m)\right| \leqslant R T_{f_{2}}$. We are trying to calculate the total change in $\arg \Phi(\mathrm{i} R ; m)$ as $R$ goes from 0 to infinity. Now

$$
\begin{aligned}
\Phi(\mathrm{i} R ; m)= & {\left[\mathrm{i} R+d_{1} m^{2}+D+a P^{*} U^{\prime}\left(N^{*}\right)\right]\left(\mathrm{i} R+d_{2} m^{2}\right)+a_{1} P^{*} U^{\prime}\left(N^{*}\right) } \\
& \times\left[C_{2}(R ; m)-\mathrm{i} S_{2}(R ; m)\right]\left\{a U\left(N^{*}\right)-\gamma_{1}\left[C_{1}(R ; m)-\mathrm{i} S_{1}(R ; m)\right]\right\} .
\end{aligned}
$$

In view of the bounds $\left|C_{j}(R ; m)\right| \leqslant 1$ and $\left|S_{j}(R ; m)\right| \leqslant 1$ for $j=1,2$ one easily concludes from expression (2.7) that, for large values of $R$,

$$
\begin{aligned}
& \operatorname{Re} \Phi(\mathrm{i} R ; m) \sim-R^{2} \\
& \operatorname{Im} \Phi(\mathrm{i} R ; m) \sim(\text { positive constant }) R
\end{aligned}
$$

Also,

$$
\begin{aligned}
\Phi(0 ; m)= & {\left[d_{1} m^{2}+D+a P^{*} U^{\prime}\left(N^{*}\right)\right] d_{2} m^{2}+a_{1} P^{*} U^{\prime}\left(N^{*}\right) C_{2}(0 ; m) } \\
& \times\left[a U\left(N^{*}\right)-\gamma_{1} C_{1}(0 ; m)\right] .
\end{aligned}
$$

But

$$
C_{2}(0 ; m)=\int_{0}^{\infty} f_{2}(t) \mathrm{e}^{-d_{1} m^{2} t} \mathrm{~d} t>0
$$


and also

$$
a U\left(N^{*}\right)-\gamma_{1} C_{1}(0 ; m) \geqslant a U\left(N^{*}\right)-\gamma_{1}=a\left(\frac{\gamma+D}{a_{1}}\right)-\gamma_{1} \geqslant \gamma+D-\gamma_{1}>0
$$

by assumption (1.11). It follows from this that $\Phi(0 ; m)>0$ for any $m=0$, $1,2, \ldots$

In view of the above facts, we see that the complex number $\Phi(\mathrm{i} R ; m)$ starts, when $R=0$, on the positive real axis, and always ends up in the second quadrant as $R \rightarrow \infty$. And the fact that $\operatorname{Re} \Phi(\mathrm{i} R ; m) \sim-R^{2}$ while the imaginary part only grows like $R$ enables us to conclude that $\lim _{R \rightarrow \infty}$ arg $\Phi(\mathrm{i} R ; m)$ must be one of the values $\pi,-\pi,-3 \pi$, etc. Fig. 1 shows some qualitative sketches of graphs that would correspond to each of these three cases. Note that only the first case is consistent with stability, since formula (2.6) then tells us that the eigenvalue equation $\Phi(\sigma ; m)=0$ has no roots in the right half of the complex plane. Any other case corresponds to having a positive (even) number of such roots. Note also that the structure of formula (2.6) also tells us that the curve traced out by $\Phi(\mathrm{i} R ; m)$ for $R \in[0, \infty)$ cannot possibly encircle the origin in the anticlockwise sense, otherwise a negative value would be yielded for the number of roots.

In obtaining theorems which are sufficient for stability of the uniform equilibrium state (2.1) we are essentially interested in finding out under what circumstances it will be true that

$$
\lim _{R \rightarrow \infty} \arg \Phi(\mathrm{i} R ; m)=\pi \quad \text { for every } m=0,1,2, \ldots
$$

While the following is not an exhaustive list of circumstances under which the above conclusion follows, we can certainly state that the conclusion holds if, for every $m=0,1,2, \ldots$, either (i) $\operatorname{Im} \Phi(\mathrm{i} R ; m)>0$ for all $R>0$, or (ii) $\operatorname{Im} \Phi(\mathrm{i} R ; m)>0$ whenever $\operatorname{Re} \Phi(\mathrm{i} R ; m)=0$. The latter works by preventing the curve $\Phi(\mathrm{i} R ; m)$ from crossing the negative imaginary axis.

Having now set up these ideas, certain theorems follow. Our first theorem; the only one we shall prove for the full two-delay model, guarantees the linear stability of (2.1) for sufficiently small mean delays.

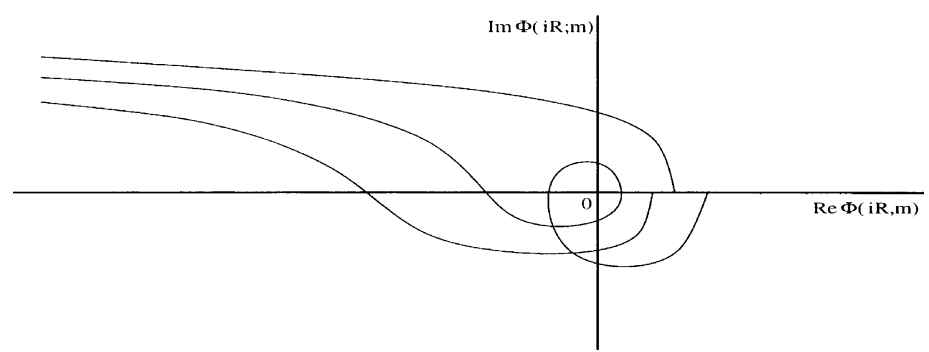

Fig. 1. Possible qualitative sketches of $\operatorname{Im} \Phi(\mathrm{i} R ; m)$ against $\operatorname{Re} \Phi(\mathrm{i} R ; m)$ for $R \in[0, \infty)$. 
Theorem 1. In the system (1.2) suppose that the parameters satisfy (1.11) and (2.2) and that the delay kernels $f_{1}$ and $f_{2}$ have mean delays $T_{f_{1}}$ and $T_{f_{2}}$ satisfying $a_{1} P^{*} U^{\prime}\left(N^{*}\right)\left(\gamma_{1} T_{f_{1}}+\left(\gamma_{1}+a U\left(N^{*}\right)\right) T_{f_{2}}\right)<D+a P^{*} U^{\prime}\left(N^{*}\right)$.

Then the steady state (2.1) of (1.2) is stable to arbitrary small perturbations.

Proof. We aim to show that $\operatorname{Im} \Phi(\mathrm{i} R ; m)>0$ for all $R>0$ and all $m=0,1,2, \ldots$ From (2.7), we have

$$
\begin{aligned}
\operatorname{Im} \Phi(\mathrm{i} R ; m)=R & {\left[d_{1} m^{2}+d_{2} m^{2}+D+a P^{*} U^{\prime}\left(N^{*}\right)\right]+a_{1} P^{*} U^{\prime}\left(N^{*}\right) } \\
\times & {\left[-a U\left(N^{*}\right) S_{2}(R ; m)+\gamma_{1} C_{2}(R ; m) S_{1}(R ; m)\right.} \\
& \left.+\gamma_{1} C_{1}(R ; m) S_{2}(R ; m)\right] .
\end{aligned}
$$

Using the various bounds on the $C_{j}(R ; m)$ and $S_{j}(R ; m)$ for $j=1,2$ we can conclude that

$$
\begin{aligned}
\operatorname{Im} \Phi(\mathrm{i} R ; m) \geqslant & R\left(D+a P^{*} U^{\prime}\left(N^{*}\right)\right)-a_{1} P^{*} U^{\prime}\left(N^{*}\right)\left[a U\left(N^{*}\right) R T_{f_{2}}+\gamma_{1} R T_{f_{1}}\right. \\
& \left.+\gamma_{1} R T_{f_{2}}\right]
\end{aligned}
$$

and this is positive for all $R>0$ under the condition on $T_{f_{1}}$ and $T_{f_{2}}$ stated in the theorem. Therefore, $\lim _{R \rightarrow \infty}$ arg $\Phi(\mathrm{i} R ; m)=\pi$ and so the eigenvalue equation $\Phi(\sigma ; m)=0$ has no roots in the right half complex plane. Since this is true for any value of the wave number $m=0,1,2, \ldots$ the result follows and the proof is complete.

\subsection{Delay in nutrient recycling only}

In this subsection we shall consider the case when the growth response of the species to nutrient uptake is instantaneous. Such a model can be considered as an approximation to the case when this particular time-scale is negligible to that on which the dead plankton decompose. This submodel can be extracted as a particular case of the general model (1.2) by setting $f_{2}(t)=\delta(t)$, the Dirac delta function, under which (1.2) reduces to

$$
\begin{aligned}
\frac{\partial N}{\partial t}= & d_{1} \frac{\partial^{2} N}{\partial x^{2}}+D\left(N^{0}-N\right)-a U(N) P \\
& +\gamma_{1} \int_{-\infty}^{t} \int_{0}^{\pi} G_{1}(x, y, t-s) f_{1}(t-s) P(y, s) \mathrm{d} y \mathrm{~d} s \\
\frac{\partial P}{\partial t}= & d_{2} \frac{\partial^{2} P}{\partial x^{2}}+P\left[-(\gamma+D)+a_{1} U(N(x, t))\right] .
\end{aligned}
$$

This can be regarded as the diffusive version of the model studied by Beretta et al. [3]. Of course, it still has the uniform steady state (2.1). The simplification of having only one delay term enables us to obtain more information on the 
stability of this steady state. Our first theorem on this is simply a corollary of Theorem 1 arising by taking $f_{2}(t)=\delta(t)$, so that $T_{f_{2}}=0$.

Theorem 2. In the system (2.9) suppose that the parameters satisfy (1.11) and (2.2) and that the delay kernel $f_{1}$ has mean delay $T_{f_{1}}$ satisfying

$$
T_{f_{1}}<\frac{D+a P^{*} U^{\prime}\left(N^{*}\right)}{a_{1} \gamma_{1} P^{*} U^{\prime}\left(N^{*}\right)} .
$$

Then the steady state (2.1) of (2.9) is stable to arbitrary small perturbations.

Our next theorem gives a condition on the various coefficients of the model and the function $U$, under which the uniform state will be stable for any normalised delay kernel $f_{1}(t)$. If the hypotheses of this theorem are satisfied then the delay is said to be harmless, meaning that it is incapable of destabilising the steady state whatever the form of the kernel $f_{1}(t)$.

Theorem 3. In the system (2.9) suppose that the parameters satisfy (1.11) and (2.2) together with

$$
\sqrt{a_{1} P^{*} U^{\prime}\left(N^{*}\right)\left(a U\left(N^{*}\right)-\gamma_{1}\right)}\left(D+a P^{*} U^{\prime}\left(N^{*}\right)\right)>a_{1} \gamma_{1} P^{*} U^{\prime}\left(N^{*}\right) .
$$

Then the steady state (2.1) of (2.9) is stable to arbitrary small perturbations.

Proof. We use the second of the two strategies enumerated earlier and aim to show that $\operatorname{Im} \Phi(\mathrm{i} R ; m)>0$ whenever $\operatorname{Re} \Phi(\mathrm{i} R ; m)=0$. We need an expression for $\Phi(\mathrm{i} R ; m)$ for our submodel. The expression is the particular case of (2.7) when $f_{2}(t)=\delta(t)$ (giving $C_{2}(R ; m)=1$ and $S_{2}(R ; m)=0$ ) so that

$$
\begin{aligned}
\Phi(\mathrm{i} R ; m)= & \left(\mathrm{i} R+d_{1} m^{2}+D+a P^{*} U^{\prime}\left(N^{*}\right)\right)\left(\mathrm{i} R+d_{2} m^{2}\right) \\
& +a_{1} P^{*} U^{\prime}\left(N^{*}\right)\left(a U\left(N^{*}\right)-\gamma_{1}\left(C_{1}(R ; m)-\mathrm{i} S_{1}(R ; m)\right)\right) .
\end{aligned}
$$

Let $R_{0}$ be a value of $R$ such that $\operatorname{Re} \Phi\left(\mathrm{i} R_{0} ; m\right)=0$. Then, from (2.10),

$$
R_{0}^{2}=d_{2} m^{2}\left(d_{1} m^{2}+D+a P^{*} U^{\prime}\left(N^{*}\right)\right)+a_{1} P^{*} U^{\prime}\left(N^{*}\right)\left(a U\left(N^{*}\right)-\gamma_{1} C_{1}\left(R_{0} ; m\right)\right)
$$

and so, since $C_{1}\left(R_{0} ; m\right) \leqslant 1$, any such value $R_{0}$ must satisfy

$$
R_{0}^{2} \geqslant a_{1} P^{*} U^{\prime}\left(N^{*}\right)\left(a U\left(N^{*}\right)-\gamma_{1}\right) .
$$

Thus

$$
\begin{aligned}
\operatorname{Im} \Phi\left(\mathrm{i} R_{0} ; m\right)= & R_{0}\left(d_{1} m^{2}+d_{2} m^{2}+D+a P^{*} U^{\prime}\left(N^{*}\right)\right) \\
& +a_{1} \gamma_{1} P^{*} U^{\prime}\left(N^{*}\right) S_{1}\left(R_{0} ; m\right) \geqslant R_{0}\left(D+a P^{*} U^{\prime}\left(N^{*}\right)\right) \\
& -a_{1} \gamma_{1} P^{*} U^{\prime}\left(N^{*}\right) \geqslant \sqrt{a_{1} P^{*} U^{\prime}\left(N^{*}\right)\left(a U\left(N^{*}\right)-\gamma_{1}\right)} \\
& \times\left(D+a P^{*} U^{\prime}\left(N^{*}\right)\right)-a_{1} \gamma_{1} P^{*} U^{\prime}\left(N^{*}\right)
\end{aligned}
$$


which is positive under the assumptions of the theorem. We conclude that $\lim _{R \rightarrow \infty} \arg \Phi(\mathrm{i} R ; m)=\pi$. This is true for all $m=0,1,2 \ldots$ and the proof is complete.

We now prove a theorem concerning the linear stability of (2.1), as a solution of (2.9), when the kernel $f_{1}$ is one of the two generic delay kernels given by the second two kernels in (1.8). In fact, we shall consider the more general family of kernels

$$
f_{1}(t)=\frac{t^{p}}{p ! \tau^{p+1}} \mathrm{e}^{-t / \tau}, \quad p=0,1,2, \ldots
$$

of which the weak and strong generic delay kernels are the particular cases $p=0$ and $p=1$. For these two cases we shall show that the uniform state (2.1) of (2.9) is linearly stable for any $\tau>0$. More generally, a condition on $p$ is needed. For this more general kernel the mean delay $T_{f_{1}}=(p+1) \tau$.

Theorem 4. In the system (2.9) suppose that the parameters satisfy (1.11) and (2.2) and that the delay kernel is given by (2.11). Suppose also that

$$
(p+1) \tan ^{-1} \tau R^{*} \leqslant \pi, \quad \text { where } R^{*}=\frac{a_{1} \gamma_{1} P^{*} U^{\prime}\left(N^{*}\right)}{D+a P^{*} U^{\prime}\left(N^{*}\right)} .
$$

Then the steady state (2.1) of (2.9) is stable to arbitrary small perturbations.

Proof. We aim to show that $\operatorname{Im} \Phi(\mathrm{i} R ; m)>0$ for all $R>0$ and all $m=0,1,2, \ldots$ Let $m$ be fixed. It is necessary to consider separately the cases $R>R^{*}$ and $0<R \leqslant R^{*}$ where $R^{*}$ is the quantity defined in the statement of the theorem. The expression for $\operatorname{Im} \Phi(\mathrm{i} R ; m)$ can be found in the proof of the previous theorem. For $R>R^{*}$, we have, using $S_{1}(R ; m) \geqslant-1$,

$$
\begin{aligned}
\operatorname{Im} \Phi(\mathrm{i} R ; m)= & R\left(d_{1} m^{2}+d_{2} m^{2}+D+a P^{*} U^{\prime}\left(N^{*}\right)\right) \\
& +a_{1} \gamma_{1} P^{*} U^{\prime}\left(N^{*}\right) S_{1}(R ; m) \geqslant R\left(D+a P^{*} U^{\prime}\left(N^{*}\right)\right) \\
& -a_{1} \gamma_{1} P^{*} U^{\prime}\left(N^{*}\right) \\
> & R^{*}\left(D+a P^{*} U^{\prime}\left(N^{*}\right)\right)-a_{1} \gamma_{1} P^{*} U^{\prime}\left(N^{*}\right)=0 .
\end{aligned}
$$

For $0<R \leqslant R^{*}$ proceed as follows. The kernel (2.11) has Laplace transform

$$
\bar{f}_{1}(\sigma)=\frac{1}{(1+\sigma \tau)^{p+1}} .
$$

Introduce $\phi(R ; m):[0, \infty) \rightarrow[0, \pi / 2)$, defined by

$$
\tan \phi(R ; m)=\frac{\tau R}{1+\tau d_{2} m^{2}}, \quad 0 \leqslant \phi(R ; m)<\pi / 2 .
$$


Then

$$
S_{1}(R ; m)=-\operatorname{Im} \bar{f}_{1}\left(\mathrm{i} R+d_{2} m^{2}\right)=\frac{\sin ((p+1) \phi(R ; m))}{\left(\left(1+\tau d_{2} m^{2}\right)^{2}+\tau^{2} R^{2}\right)^{(p+1) / 2}} .
$$

Now, if $0<R \leqslant R^{*}$ then, since $\phi(R ; m)$ is an increasing function of $R$,

$$
\phi(R ; m) \leqslant \tan ^{-1}\left(\frac{\tau R^{*}}{1+\tau d_{2} m^{2}}\right) .
$$

Hence

$$
(p+1) \phi(R ; m) \leqslant(p+1) \tan ^{-1}\left(\frac{\tau R^{*}}{1+\tau d_{2} m^{2}}\right) \leqslant(p+1) \tan ^{-1} \tau R^{*} \leqslant \pi
$$

by hypothesis. It follows that $S_{1}(R ; m) \geqslant 0$ and hence, also, $\operatorname{Im} \Phi(\mathrm{i} R ; m)>0$ for $0<R \leqslant R^{*}$ as desired. This all holds independently of the wave number $m$ and so the proof is complete.

Remark. Since $\tan ^{-1} \tau R^{*}<\pi / 2$ the hypotheses of the theorem are obviously satisfied when $p=0$ and 1 . It follows that for the weak and strong generic delay kernels we have stability independently of $\tau$.

\subsection{Delay in growth response to nutrient uptake}

The second submodel we shall consider is the case when the delay involved in the decomposition of the dead plankton is zero, or very small compared to the delay in the growth response of the plankton to nutrient uptake. This submodel is a particular case of the general model when $f_{1}(t)=\delta(t)$. The model (1.2) becomes, in this case,

$$
\begin{aligned}
& \frac{\partial N}{\partial t}=d_{1} \frac{\partial^{2} N}{\partial x^{2}}+D\left(N^{0}-N\right)-a U(N) P+\gamma_{1} P(x, t), \\
& \frac{\partial P}{\partial t}=d_{2} \frac{\partial^{2} P}{\partial x^{2}}+P\left[-(\gamma+D)+a_{1} \int_{-\infty}^{t} \int_{0}^{\pi} G_{2}(x, y, t-s) f_{2}(t-s) U(N(y, s)) \mathrm{d} y \mathrm{~d} s\right] .
\end{aligned}
$$

In this section we shall demonstrate that the equilibrium (2.1) can be driven unstable for sufficiently small diffusivities $d_{1}, d_{2}$ when the delay kernel $f_{2}(t)$ assumes the form

$$
f_{2}(t)=\frac{1}{\tau} \mathrm{e}^{-t / \tau}
$$

the Laplace transform of which is $\bar{f}_{2}(s)=1 /(1+s \tau)$. In this case the general eigenvalue equation (2.5) can be rearranged after some algebra into a cubic equation for $\sigma$ :

$$
\sigma^{3}+a_{1}(\tau, m) \sigma^{2}+a_{2}(\tau, m) \sigma+a_{3}(\tau, m)=0
$$


with coefficients $a_{i}(\tau, m)$ given by

$$
\begin{aligned}
a_{1}(\tau, m)= & \frac{1}{\tau}+\left(2 d_{1}+d_{2}\right) m^{2}+D+a P^{*} U^{\prime}\left(N^{*}\right), \\
a_{2}(\tau, m)= & d_{1}\left(d_{1}+2 d_{2}\right) m^{4}+\left(d_{1}+d_{2}\right) \\
& \times\left(\frac{1}{\tau}+D+a P^{*} U^{\prime}\left(N^{*}\right)\right) m^{2}+\frac{1}{\tau}\left(D+a P^{*} U^{\prime}\left(N^{*}\right)\right), \\
a_{3}(\tau, m)= & d_{2} m^{2}\left(\frac{1}{\tau}+d_{1} m^{2}\right)\left(d_{1} m^{2}+D+a P^{*} U^{\prime}\left(N^{*}\right)\right) \\
& +\frac{1}{\tau} a_{1} P^{*} U^{\prime}\left(N^{*}\right)\left(a U\left(N^{*}\right)-\gamma_{1}\right) .
\end{aligned}
$$

Note that all three of these coefficients are positive for all parameter combinations. If, additionally,

$$
a_{1}(\tau, m) a_{2}(\tau, m)>a_{3}(\tau, m), \quad \text { for all } m=0,1,2, \ldots,
$$

then, by the Routh Hurwitz criteria, the equilibrium (2.1) is locally stable. We can demonstrate that the equilibrium can be driven unstable for sufficiently small diffusivities under a further condition on the parameters. To keep the algebra as simple as possible we let $m=0$; continuity arguments imply that the same general conclusions will hold for any given $m \neq 0$ provide the diffusivities are both sufficiently small. Thinking of the delay $\tau$ as bifurcation parameter, we may anticipate a Hopf bifurcation (implying the appearance of time-periodic oscillations) if there exists some value of $\tau$ for which

$$
a_{1}(\tau, 0) a_{2}(\tau, 0)=a_{3}(\tau, 0) .
$$

If this is so then (2.13) has a pair of purely imaginary roots $\sigma= \pm i \omega$ where $\omega>0$ satisfies

$$
\omega^{2}=a_{2}(\tau, 0)=\frac{1}{\tau}\left(D+a P^{*} U^{\prime}\left(N^{*}\right)\right) .
$$

Eq. (2.14) yields that $\tau$ satisfies

$$
\frac{1}{\tau}\left(D+a P^{*} U^{\prime}\left(N^{*}\right)\right)=a_{1} P^{*} U^{\prime}\left(N^{*}\right)\left(a U\left(N^{*}\right)-\gamma_{1}\right)-\left(D+a P^{*} U^{\prime}\left(N^{*}\right)\right)^{2}
$$

and thus a Hopf bifurcation occurs if and only if

$$
a_{1} P^{*} U^{\prime}\left(N^{*}\right)\left(a U\left(N^{*}\right)-\gamma_{1}\right)>\left(D+a P^{*} U^{\prime}\left(N^{*}\right)\right)^{2} .
$$

It is not immediately clear that $(2.17)$ can be satisfied by any parameter combination especially since $N^{*}$ and $P^{*}$ depend on the other parameters and since conditions (1.11) and (2.2) must hold. Therefore, to investigate further whether (2.17) can hold we shall work with the Michaelis-Menten function 
$U(N)=N /(k+N)$ and compute the equilibrium components $\left(N^{*}, P^{*}\right)$ explicitly. For this choice of the uptake function $U(N)$, inequality (2.17) can be put into the form

$$
\begin{aligned}
& \frac{\left(a_{1}-\gamma-D\right)^{2}}{k a_{1}}\left(N^{0}-\frac{k(\gamma+D)}{a_{1}-\gamma-D}\right) \\
& >D\left(1+\frac{a\left(a_{1}-\gamma-D\right)^{2}\left(N^{0}-\frac{k(\gamma+D)}{a_{1}-\gamma-D}\right)}{k a_{1}^{2}\left(\frac{a}{a_{1}}(\gamma+D)-\gamma_{1}\right)}\right)^{2} .
\end{aligned}
$$

It is clear that, all other parameters being fixed, inequality (2.18) will be satisfied for $D$ sufficiently small. The value of $\tau$ at which the bifurcation occurs is then given by (2.16).

The algebra is considerably more difficult for $m>0$. The reader will find, for example, that the equation for $\tau$ no longer has the structure of (2.16) but becomes instead a quadratic equation. However by continuity arguments we can still anticipate the same general conclusions holding if $d_{1}$ and $d_{2}$ are both sufficiently small. Note, however, that for all $m$ sufficiently large (or $m>0$ fixed and $d_{1}, d_{2}$ both sufficiently large) we necessarily have

$$
a_{1}(\tau, m) a_{2}(\tau, m)>a_{3}(\tau, m)
$$

and thus large diffusivities are stabilising.

In the remainder of this subsection we present the results of some numerical simulations of the system (2.12). The simulations were carried out for the case when $f_{2}(t)=(1 / \tau) \mathrm{e}^{-t / \tau}$. The use of this particular delay kernel enables us to reformulate system (2.12) as a reaction-diffusion system without delay terms (the delay parameter $\tau$ appears as a coefficient in the reformulated system). This is particularly useful for the purposes of numerical simulation. Define

$$
Q(x, t)=\int_{-\infty}^{t} \int_{0}^{\pi} G_{2}(x, y, t-s) \frac{1}{\tau} \mathrm{e}^{-(t-s) / \tau} U(N(y, s)) \mathrm{d} y \mathrm{~d} s .
$$

Next, note that although $G_{2}(x, y, t)$ is defined by being the solution of

$$
\frac{\partial G_{2}}{\partial t}=d_{1} \frac{\partial^{2} G_{2}}{\partial y^{2}}, \quad \frac{\partial G_{2}}{\partial y}=0 \quad \text { at } y=0, \pi, \quad G_{2}(x, y, 0)=\delta(x-y),
$$

it actually also satisfies

$$
\frac{\partial G_{2}}{\partial t}=d_{1} \frac{\partial^{2} G_{2}}{\partial x^{2}}, \quad \frac{\partial G_{2}}{\partial x}=0 \quad \text { at } x=0, \pi, \quad G_{2}(x, y, 0)=\delta(x-y) .
$$

In view of this fact, if we differentiate (2.19) with respect to $t$ the result is

$$
\frac{\partial Q}{\partial t}=d_{1} \frac{\partial^{2} Q}{\partial x^{2}}+\frac{1}{\tau}(U(N)-Q)
$$


It is also easily seen that $\partial Q / \partial x=0$ at $x=0, \pi$. In view of these facts, we assert that system $(2.12)$, in the case when $f_{2}(t)=(1 / \tau) \mathrm{e}^{-t / \tau}$, can be replaced by

$$
\begin{aligned}
& \frac{\partial N}{\partial t}=d_{1} \frac{\partial^{2} N}{\partial x^{2}}+D\left(N^{0}-N\right)-a U(N) P+\gamma_{1} P(x, t), \\
& \frac{\partial P}{\partial t}=d_{2} \frac{\partial^{2} P}{\partial x^{2}}+P\left(-(\gamma+D)+a_{1} Q\right), \\
& \frac{\partial Q}{\partial t}=d_{1} \frac{\partial^{2} Q}{\partial x^{2}}+\frac{1}{\tau}(U(N)-Q)
\end{aligned}
$$

with homogeneous Neumann boundary conditions for each component. In fact, the solution set of (2.12) is not quite the same as that of (2.20). Indeed, (2.19) requires that the $N$ and $Q$ components of the initial data be related by

$$
Q(x, 0)=\int_{-\infty}^{0} \int_{0}^{\pi} G_{2}(x, y,-s) \frac{1}{\tau} \mathrm{e}^{s / \tau} U(N(y, s)) \mathrm{d} y \mathrm{~d} s
$$

so the two systems are equivalent only for initial data such that (2.21) holds. However, if we solve the third equation of (2.20) independently of the others the result is

$$
Q(x, t)=\widetilde{Q}(x, t)+\int_{-\infty}^{t} \int_{0}^{\pi} G_{2}(x, y, t-s) \frac{1}{\tau} \mathrm{e}^{-(t-s) / \tau} U(N(y, s)) \mathrm{d} y \mathrm{~d} s,
$$

where $\widetilde{Q}(x, t)$ satisfies

$$
\frac{\partial \widetilde{Q}}{\partial t}=d_{1} \frac{\partial^{2} \widetilde{Q}}{\partial x^{2}}-\frac{1}{\tau} \widetilde{Q}, \quad \widetilde{Q}_{x}=0 \text { at } x=0, \pi
$$

so that $\widetilde{Q}(x, t) \rightarrow 0$ as $t \rightarrow \infty$. Therefore, we are assured that the use of initial data not satisfying (2.21) has only a transient effect on the solution dynamics. This is important since $Q(x, 0)$ cannot in practice be calculated from (2.21) for any non-homogeneous initial data, even in the simplest reasonable case when $U(N)=N /(k+N)$. Our initial data were always taken to be small sinusoidal perturbations from the equilibrium values; in this way (2.21) will be approximately satisfied.

Our numerical simulations were carried out using the NAG routine D03PCF. The Michaelis-Menten function $U(N)=N /(k+N)$ was used. The parameter values are taken from [13] and are: $k=5.85, D=0.08, N^{0}=3.66$, $a=4.25, a_{1}=3.45, \gamma=0.58$ and $\gamma_{1}=0.12$. The values for the delay $\tau$ and the diffusivities are shown in the captions. Only the solution for the plankton, $P(x, t)$, is plotted. For these parameter values the equilibrium $P^{*}=0.263$, and numerical computation indicates that inequality (2.18) is satisfied for $0<$ $D<0.15955$. Our value of $D$ being within this range, a Hopf bifurcation can be anticipated as $\tau$ is increased, and (2.16) predicts this will occur at $\tau=7.245$. The simulation shown in Fig. 2 is for $\tau=2$, showing rapid convergence of 


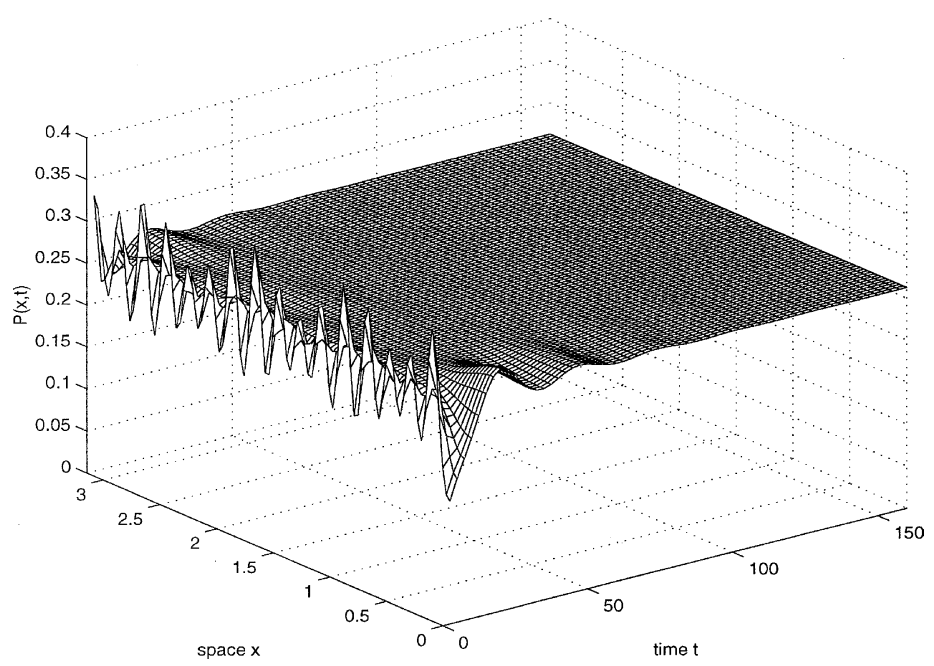

Fig. 2. Numerical simulation of system (2.20) under homogeneous Neumann boundary conditions. In this simulation the delay $\tau=2$ and we chose $d_{1}=0.001$ and $d_{2}=0.002$. Values for the other parameters are stated in the text. Evolution of $P(x, t)$ is to the uniform steady state $P^{*}=0.263$.

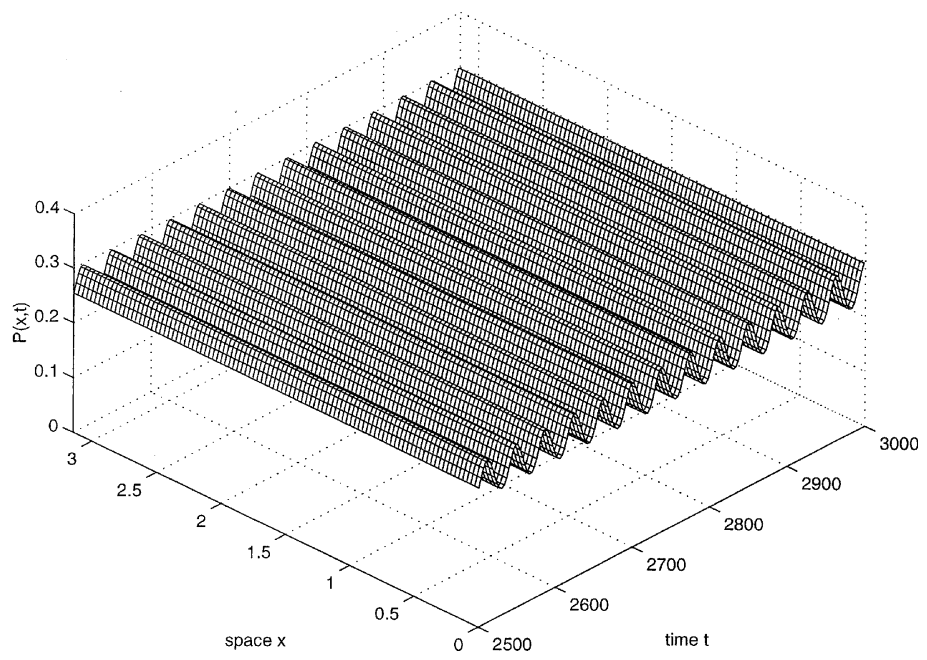

Fig. 3. Numerical simulation of system (2.20) under homogeneous Neumann boundary conditions. In this simulation, $\tau=7.4, d_{1}=0.1$ and $d_{2}=0.2$. Evolution is to a time periodic, spatially uniform solution.

$P(x, t)$ to the equilibrium $P^{*}$. The second simulation (shown without initial transients in Fig. 3) is for $\tau=7.4$ and shows a purely time-periodic solution 
convergence toward which was slow. At the bifurcation the periodic solution will have period $2 \pi / \omega$, with $\omega$ given by (2.15) and $\tau$ by (2.16), yielding a figure for the parameter values we have used of about 37.367 at bifurcation. Our value of $\tau$ is slightly beyond the bifurcation. The numerically computed solution seems to have a period of about 38.5, suggesting a gradual increase in the period as $\tau$ is increased beyond the bifurcation.

\section{Discussion}

This paper has addressed the issue, in the context of a diffusive nutrientplankton model, of how to correctly incorporate time-delay effects into a mathematical model of a diffusing population. This modelling issue is now reasonably well understood for the case when the domain is infinite. Therefore in this paper we have been concentrating on the more difficult case of a finite spatial domain, which introduces additional difficulties in that interactions with the domain's boundaries during the time-delay period, when the individuals are drifting to their current position from where they were previously, must be taken into account in deriving the time delay terms. As a consequence of this we find that the spatial averaging kernels are more complicated than for the infinite domain case, but for a one-dimensional domain may still be computed explicitly and in the homogeneous Neumann problem are Fourier cosine series. This enables considerable analytic progress to be made in that dispersion relations can be found for the linear stability problem, and that therefore the linear stability analysis and local bifurcation structure can be computed analytically. In principle it should be possible to extend the ideas to $n$-dimensional domains, especially since the heat equation can be solved explicitly in many geometries.

An interesting and worthwhile, but highly non-trivial question remaining to be addressed, is the extent to which these ideas can be carried over to other kinds of diffusion. Of course, the kernels $G_{1}$ and $G_{2}$ will not be found by solving the heat equation, they will be found by solving whatever diffusion equations are describing the drift.

For the full general model (with both delays present) we showed that, under certain reasonable assumptions on the parameters, if the mean delays are relatively small, then the steady state of the model is stable under arbitrary small perturbations. This agrees with the analyses on the plankton models with local delays $[9,10]$.

To study the effects of the two delays separately, we have considered two special cases: when delay is present only in the nutrient recycling term, and when delay is present only in the growth response term. We have shown that the first of these submodels is always stable if the average delay is relatively small, that under certain conditions the delays are completely incapable of 
destabilising the interior equilibrium and, rather interestingly, that the frequently used weak and strong generic delay kernels are not capable of destabilising this equilibrium state, for all ecologically relevant values of the other parameters. This phenomenon has also been observed in plankton models with local delays [6]. For our second submodel, with delay only in the growth response term, we have shown that as the mean delay is increased a Hopf bifurcation is possible to a time-periodic solution. Although our detailed calculations were carried out only for zero diffusivities, or zero wave number perturbation, continuity arguments mean that the Hopf bifurcation will also occur for any non-zero wave number $m=1,2,3, \ldots$ provided the diffusivities are both sufficiently small. These Hopf bifurcations are to spatially uniform temporally periodic solutions (when $m=0$ ) and to standing wave solutions (when $m>0$ ). Numerical simulations confirm the analytical results and also suggest that, in this particular model, purely temporal oscillations are the kind most likely to be observed. Our analysis of the two submodels also suggests that the delay in the growth response to nutrient uptake is much more likely to be destabilising than the delay in nutrient recycling.

The system (1.1) was shown by Ruan [13] to possess non-constant stationary solutions through the mechanism of diffusion-driven instability. Such solutions do not seem to occur in the spatio-temporal delay model of the present paper, at least not via bifurcation from a spatially uniform equilibrium. Thus it would appear that the inclusion of spatial averaging in this system is in some sense restricting the types of possible solutions, possibly due to the averaging playing some kind of homogenising role. Of course, temporally periodic solutions without spatial structure are not affected by spatial averaging. These observations may be useful in explaining and understanding planktonic patchiness [12].

\section{References}

[1] R.M. Nisbet, W.S.C. Gurney, Model of material cycling in a closed ecosystem, Nature 264 (1976) 633-635.

[2] R.H. Whittaker, Communities and Ecosystems, Macmillan, New York, 1975.

[3] E. Beretta, G.I. Bischi, F. Solimano, Stability in chemostat equations with delayed nutrient recycling, J. Math. Biol. 28 (1990) 99-111.

[4] G.I. Bischi, Effects of time lags on transient characteristics of a nutrient cycling model, Math. Biosci. 109 (1992) 151-175.

[5] J. Caperon, Time lag in population growth response of isochrysis galbana to a variable nitrate environment, Ecology 50 (1969) 188-192.

[6] S. Ruan, The effect of delays on stability and persistence in plankton models, Non-linear Anal. Theor. Meth. Applicat. 24 (1995) 575-585.

[7] E. Beretta, Y. Takeuchi, Qualitative properties of chemostat equations with time delays: boundedness, local and global asymptotic stability, Diff. Eqns. Dyn. Syst. 2 (1994) 1940. 
[8] E. Beretta, Y. Takeuchi, Qualitative properties of chemostat equations with time delays II, Diff. Eqns. Dyn. Syst. 2 (1994) 263-288.

[9] X.-Z. He, S. Ruan, Global stability in chemostat-type plankton models with delayed nutrient recycling, J. Math. Biol. 37 (1998) 253-271.

[10] X.-Z. He, S. Ruan, H. Xia, Global stability in chemostat-type equations with distributed delays, SIAM J. Math. Anal. 29 (1998) 681-696.

[11] A. Okubo, Diffusion and Ecological Problems: Mathematical Models, Springer-Verlag, Berlin, 1980.

[12] S.A. Levin, L.A. Segel, Hypothesis for the origin of planktonic patchiness, Nature 259 (1976) 259.

[13] S. Ruan, Turing instability and travelling waves in diffusive plankton models with delayed nutrient recycling, IMA. J. Appl. Maths. 61 (1998) 15-32.

[14] N.F. Britton, Spatial structures and periodic travelling waves in an integro-differential reaction-diffusion population model, SIAM J. Appl. Math. 50 (1990) 1663-1688.

[15] S.A. Gourley, N.F. Britton, A predator prey reaction-diffusion system with non-local effects, J. Math. Biol. 34 (1996) 297-333.

[16] H.R. Thieme, X.-Q. Zhao, A non-local delayed and diffusive predator-prey model, Non-linear Anal.: Real World Applicat. 2 (2001) 145-160.

[17] N. Madras, J. Wu, X. Zou, Local-non-local interaction and spatial-temporal patterns in single species population over a patchy environment, Canad. Appl. Math. Quart. 4 (1996) 109-134.

[18] J.W.-H. So, J. Wu, X. Zou, Structured population on two patches: modeling dispersal and delay, J. Math. Biol. 43 (2001) 37-51.

[19] K. Boushaba, S. Ruan, Instability in diffusive ecological models with non-local delay effects, J. Math. Anal. Appl. 258 (2001) 269-286.

[20] N. MacDonald, Time Lags in Biological Models, Lecture Notes in Biomathematics, vol. 27, Springer-Verlag, Berlin, 1978.

[21] S.A. Gourley, M.V. Bartuccelli, Parameter domains for instability of uniform states in systems with many delays, J.Math. Biol. 35 (1997) 843-867. 